\title{
Aspectos nutricionais e econômicos da dieta vegetariana: uma análise usando a Programação Linear
}

\author{
Luciana Yoshie Tsuchiya ${ }^{1}$ \\ IFPR, Paranavaí, PR \\ Cecília Osteti Furtado ${ }^{2}$ \\ IFPR, Paranavaí, PR
}

\begin{abstract}
Resumo. Nos últimos anos, têm ocorrido uma crescente adesão ao vegetarianismo. De acordo com uma pesquisa do IBOPE inteligência de abril de 2018, $14 \%$ da população brasileira se declara vegetariana. $\mathrm{O}$ crescimento surpreendente de pessoas que excluem de sua dieta alimentos de origem animal reflete tendências mundiais por uma busca de uma alimentação que seja mais saudável, sustentável e mais ética. Porém, quando se fala de dieta vegetariana duas dúvidas são frequentes. É possível obter uma dieta vegetariana que supra as recomendações nutricionais para o bom funcionamento do organismo? A dieta vegetariana é uma dieta com custo alto? A programação linear pode ajudar a responder essas duas questões, ou seja, determinar uma dieta vegetariana que atenda a recomendações nutricionais específicas, ao mesmo tempo que otimiza o seu custo. O problema da dieta é um problema clássico da programação linear, originalmente utilizado para determinar uma dieta onívora com menor custo. Nesse trabalho o modelo clássico foi adaptado para determinar uma dieta vegetariana balanceada de um dia com custo mínimo. Para a dieta vegetariana é preciso levar em consideração quantidades diferenciadas e combinações de alimentos que favorecem ou desfavorecem a biodisponibilidade de nutrientes. Na modelagem do problema, procuramos incorparar algumas dessas questões. Para tanto, foi preciso modelar o problema como um problema de programação linear inteira. Trabalhamos com três tipos de dietas vegetarianas para uma pessoa adulta entre 19 e 50 anos do sexo feminino. Os resultados foram satisfatórios, mostrando que é possível atender as recomendações nutricionais específicas da dieta, bem como obter dietas com custo acessível.
\end{abstract}

Palavras-chave. Biodisponibilidade, Programação Linear Inteira, Otimização, Problema da Dieta.

\section{Introdução}

De acordo com a Sociedade Vegetariana Brasileira, é considerado vegetariano o indivíduo que exclui de sua alimentação todos os tipos de carne, incluindo seus derivados, podendo ou não utilizar laticínios ou ovos.

O vegetariano ainda pode ser classificado com diferentes terminologias de acordo com a inclusão ou não dos derivados animais à sua dieta. Ovolactovegetarianos é o vegetariano que inclui ovos, leite e laticínios em sua dieta alimentar. Lactovegetarianos é o vegetariano que inclui em sua dieta apenas o leite e laticínios, não fazendo uso de ovos. Vegetariano estrito (ou puro) é o vegetariano que não utiliza nenhum derivado animal na sua alimentação.

No que diz respeito a saúde, durante as últimas décadas, estudos têm mostrado os benefícios do vegetarianismo e de outras dietas baseadas em alimentos vegetais para a saúde humana. Grandes

\footnotetext{
${ }^{1}$ luciana.tsuchiya@ifpr.edu.br

2 ce.cifurtado1000@gmail.com
} 
organizações, como a Organização Mundial de Saúde (OMS), têm se manifestado sobre os riscos do consumo elevado de carnes. A ingestão de carne tem sido relacionada ao aumento do risco de uma série de doenças crônicas não-transmissíveis, como alguns cânceres e doença cardiovasculares, ao passo que o abundante consumo de alimentos essenciais da dieta vegetariana, como leguminosas, hortaliças, frutas, alimentos não refinados e castanhas, têm sido constantemente associado a um menor risco de contração dessas doenças [6], além de maior controle do peso corporal, maior controle da insulina e menor risco de incidência de cancro [4].

Além da preocupação com a saúde, outros motivos que têm levado as pessoas a aderirem à dieta vegetariana são a religião, a economia, a preocupação com o impacto ambiental negativo da pecuária e a indignação com as condições de vida cruéis impostas aos animais usados nos processos de produção [4].

Segundo Couceiro, et al. [2], a dieta vegetariana difere da dieta onívora, que inclui carnes e vegetais, em aspectos que vão além da simples exclusão da carne. Os vegetarianos fazem um consumo elevado de vegetais, frutas, cereais, legumes e castanhas, além de sua dieta conter menos quantidade de gordura saturada e maior quantidade de gorduras insaturada, carboidratos e fibras.

Porém quando se fala de dietas vegetarianas duas dúvidas ainda são frequentes:

1) A dieta vegetariana supre as necessidades nutricionais do organismo?

2) A dieta vegetariana é uma dieta com custo alto?

Não raro, vegetarianos são questionados sobre como fazem para substituir a proteína. E é comum as pessoas relacionarem a dieta vegetariana à alimentos especiais, o que a tornaria uma dieta de custo alto.

Nesse trabalho utilizamos a programação linear para analisar essas duas questões. Adaptamos o modelo clássico da dieta na programação linear para obter dietas vegetarianas balanceadas de custo mínimo.

Para a dieta vegetariana há algumas indicações diferenciadas de quantidades de nutrientes que devem ser ingeridos, bem como considerações a respeito da biodisponibilidade de nutrientes dos alimentos. A biodisponibilidade diz respeito a quanto o organismo absorverá e utilizará dos nutrientes disponíveis nos alimentos e ela pode aumentar ou diminuir de acordo com as interações entre os nutrientes contidos nos alimentos ingeridos. Sua aplicabilidade às dietas vegetarianas é fundamental devido às características especiais deste padrão de dieta. Procuramos incorporar à modelagem do problema algumas restrições que levam em conta a biodisponibildiade dos nutrientes na dieta vegetariana.

\section{O Problema da dieta}

O problema da Dieta é um dos problemas clássicos da programação linear. O objetivo do problema original era determinar uma dieta (onívora) de menor custo que satisfaça às restrições mínimas de nutrientes de uma dieta balanceada. Em sua formulação matemática original são considerados $n$ alimentos que podem fazer parte uma da dieta. Cada um possui um determinado custo unitário $c_{j}, j=1, \ldots, n$ e uma quantidade de $m$ nutrientes relevantes para a dieta. Desejava-se então, obter uma dieta adequada, que atendesse a uma quantidade mínima $b_{i}, i=1, \ldots, m$ de cada nutriente, com um custo mínimo. Dessa forma, o modelo matemático original da dieta é dado por:

$$
\begin{gathered}
\min \\
\text { sujeito a: }\left\{\begin{array}{l}
c_{1} x_{1}+c_{2} x_{2}+\cdots c_{n} x_{n} \\
a_{11} x_{1}+a_{12} x_{2}+\cdot+a_{1 n} x_{n} \geq b_{1} \\
\vdots \\
a_{m 1} x_{1}+a_{m 2} x_{2}+\cdot+a_{m n} x_{n} \geq b_{m} \\
x_{j} \geq 0, \quad j=1, \ldots, n,
\end{array}\right.
\end{gathered}
$$


em que $x_{j}$, para $j=1, \ldots, n$, são as variáveis do problema e representam a quantidade de alimento $j$ que deve entrar na dieta. $j=1, \ldots, n$.

Dantzig, em 1947, resolveu o problema da dieta utilizando pela primeira vez o método Simplex [3], mas segundo Dooren (2018), somente a partir de 2003 é que se intensificou a aplicação da programação linear às questões relacionadas à nutrição humana. Em uma revisão com base na literatura mais recente (entre 2000 a 2014) [9], Van Dooren constatou que posteriormente às restrições nutricionais e de custos, surgiram também, problemas com restrições de aceitabilidade e restrições ecológicas. Além disso, verificou que a programação linear pode ser aplicada a uma variedade de problemas alimentares, como programas nacionais de alimentação, guias dietéticos e dietas que contemplem questões individuais.

\section{Recomendações nutricionais da dieta vegetariana}

De acordo com a bibliografia sobre dietas vegetarianas alguns nutrientes merecem atenção nesse tipo de dieta $[2,7]$, a saber:

- Ômega-3 : Para que o Ômega $3(\Omega-3)$ presente nos vegetais possa ser utilizado pelo organismo, ele precisa ser convertido nas formas ativas (EPA - ácido eicosapentaenoico e DHA ácido docosahexaenoico), ao contrário do Ômega 3 encontrado nos peixes e em alguns poucos alimentos de origem animal que já possuem esses fatores. O que ocorre é que o Ômega-6 ( $\Omega$ $6)$ e Ômega-3 $(\Omega-3)$ provenientes de vegetais competem pelas mesmas enzimas do organismo que os deixam prontos para ação, por isso a OMS juntamente com a Organização das Nações Unidas para Agricultura e Alimentação (FAO) recomendam que a razão entre a ingestão de $\Omega-6$ e $\Omega$-3 seja de $5: 1$, sendo aceitável até $10: 1$. As DRIs - Dietary Reference Intakes [5], também determinam que na dieta vegetariana deve-se consumir o dobro da quantidade de $\Omega-3$ das dietas onívoras, conforme a tabela 1 .

- Proteínas (macronutriente): Estudos que avaliam as necessidades protéicas não confirmam a recomendação de ingestão de quantidades diferentes de proteínas entre vegetarianos e não vegetarianos e que além disso, no organismo humano a incorporação da proteína vegetal não é diferente da animal [5]. Contrariando o senso comum, estudos mostram que mesmo sem ovos e laticínios, a proteína vegetal é suficiente para atender à necessidade humana de aminoácidos.

- Ferro: Como a biodisponibilidade média de ferro na dieta vegetariana é baixa, as DRIs indicam para essa dieta o dobro da quantidade de ferro indicado para a dieta onívora (veja 1). Ainda assim, é importante adequar a dieta de acordo com os fatores que otimizam e inibem a absorção do ferro (não-heme). O cálcio por exemplo, diminui a absorção do ferro pelo organismo, logo é recomendável evitar consumir alimentos ricos em ferro e cálcio na mesma refeição.

- Zinco: A carne é uma boa fonte de zinco, assim ao suprimí-la da dieta deve-se prestar atenção maior em relação a outras fontes de alimentos que contém zinco. As DRIs indicam para a dieta vegetariana a ingestão de $50 \%$ a mais que quantidade de zinco indicada para a dieta onívora (tabela 1). Além disso, é importante adequadar a dieta a fatores que otimizam ou inibim a sua absorção. A vitamina $\mathrm{C}$ por exemplo, ajuda a melhorar a absorção do zinco.

- Cálcio: Os leites e seus derivados são a maior fonte usual de cálcio para a maioria das pessoas, por isso apenas na dieta vegetariana estrita (sem consumo de ovos e laticínios) deve-se prestar mais atenção à escolha de outros alimentos que sejam fontes de cálcio. 
- Vitamina B12: O consumo de B12 não costuma ser um problema para vegetarianos que consomem ovos e laticínios regularmente, pois estes alimentos são fontes dessa vitamina. Para os vegetarianos estritos há a necessidade de complementação.

- Vitamina C: A ingestão de vitamina C ajuda a aumentar a biodisponibilidade do ferro e do cálcio. O uso de $75 \mathrm{mg}$ por exemplo, aumenta a absorção de ferro em 3 a 4 vezes. Por isso incluímos este nutriente na modelagem do problema.

Em relação aos macronutrientes proteína, carboidrato e gordura, os valores máximos e mínimos da tabela 1 foram obtidos considerando a ingestão média diária de 2.000 quilocalorias (kcal) adotada pelo Guia Alimentar para a População Brasileira [1] e o percentual calórico de cada macronutriente indicado pelas DRIs, considerando que 1 grama de proteína e 1 grama de carboidrato, são equivalentes a 4 calorias cada um e 1 grama de gordura é equivalente a 9 calorias.

Tabela 1: Quantidade de ingestão diária de nutrientes recomendada para pessoa do sexo feminino de 19 a 50 anos

\begin{tabular}{|c|c|c|c|c|c|c|c|c|c|c|}
\hline Nutrientes & & $\Omega \mathbf{3}$ & Prot. & Carb. & Gord. & Ferro & Zinco & Cálcio & B12 & Vit.C \\
\hline Vegeta- & Máx & 2,2 & 50 & 225 & 55,56 & 36 & 12 & 1000 & 2,4 & 60 \\
\hline rianos & Min & - & 175 & 325 & 77,78 & - & - & - & - & - \\
\hline Onívoros & & 1,1 & igual & igual & igual & 18 & 8 & igual & igual & igual \\
\hline
\end{tabular}

Na tabela 1 a quantidade de Ômega - 3, de macronutrientes, Zinco, Cálcio e Vitamina C estão em gramas, a quantidade de Ferro está em miligramas e a quantidade de B12 está em microgramas.

\section{Modelagem do problema de programação linear da dieta vegetariana}

Para que fosse possível incorporar questões de biodisponibilidade na modelagem do problema, limitar a quantidade de alimentos consumida em cada refeição, bem como limitar a quantidade de um mesmo tipo de alimento, optamos por trabalhar com porções inteiras de alimentos e portanto o problema foi modelado como um problema de programação linear inteira.

Consideramos na modelagem matemática 6 refeições: café da manhã $(\mathrm{CM})$, lanche da manhã $(\mathrm{LN})$, almoço (A), café da tarde (CT), jantar (J) e lanche da noite (LN).

Para compor a dieta vegetariana foram selecionados 143 alimentos da Tabela Brasileira de Composição de Alimentos - TACO, [8]. A tabela contém as quantidades de nutrientes e calorias para 100 gramas de cada alimento e portanto as quantidades de nutrientes tiveram que ser adaptadas para porção. Consideramos os 9 nutrientes da tabela 1 ordenados como na tabela e ainda o Ômega 6 .

O custo dos alimentos foram obtidos na internet a partir de sites de supermecados da região do Noroeste do Paraná no mês de setembro de 2019.

Os dados do problema são os seguintes:

- Variáveis de decisão: $X_{C M}, X_{L M}, X_{A}, X_{L T}, X_{J}, X_{L N}$ são vetores com 143 entradas. Cada vetor representa uma das refeições e cada entrada representa um alimento. A entrada $k$ assumir o valor 1 se o alimento $k$ entrar na respectiva refeição ou o valor 0 se o alimento $k$ não entrar.

- $c=\left[c_{1}, c_{2}, \ldots, c_{143}\right]$ é o vetor de custos em que $c_{k}$ é o custo de uma porção do alimento $k$.

- $b_{i n f}=\left[b_{i n f_{1}}, b_{i n f_{2}}, \ldots, b_{i n f_{9}}\right]$ é o vetor com as quantidades mímima dos nove nutrientes da tabela 1 , necessárias para uma dieta vegetariana balanceada. 
- $b_{\text {sup }}=\left[b_{\text {sup }_{1}}, b_{\text {sup }_{2}}, \ldots, b_{\text {sup }_{9}}\right]$ é o vetor com a quantidade máxima recomendada dos nove nutrientes da tabela 1. Quando não há restrição superior, a entrada correspondente de $b_{\text {sup }}$ tem um valor muito grande.

- $A=\left(a_{i k}\right)_{9 \times 143}$ : matriz em que cada entrada $a_{i k}$ representa a quantidade do nutriente $i$ em uma porção do alimento $k$. Denotaremos a linha $i$ da matriz $A$ por $A_{i}$.

- $O 6=\left[O 6_{1}, O 6_{2}, \ldots, O 6_{143}\right]$ é um vetor em que cada entrada $O 6_{k}$ representa a quantidade de Ômega 6 em uma porção do alimento $k$.

- $\mathcal{F}$ e $\mathcal{C}$ são os conjuntos dos índices dos alimentos ricos em ferro e cálcio, respectivamente.

A problema de programação linear da dieta vegetariana ficou da seguinte forma:

$$
\begin{aligned}
& \min \left\{c^{T} X_{C M}+c^{T} X_{L M}+c^{T} X_{A}+c^{T} X_{C T}+c^{T} X_{J}+c^{T} X_{N}\right\} \\
& \text { sujeito a: } \\
& A \cdot X_{C M}+A \cdot X_{L M}+A \cdot X_{A}+A \cdot X_{C T}+A \cdot X_{J}+A \cdot X_{N} \geq b_{i n f} \\
& A \cdot X_{C M}+A \cdot X_{L M}+A \cdot X_{A}+A \cdot X_{C T}+A \cdot X_{J}+A \cdot X_{N} \leq b_{\text {sup }} \\
& 06 \cdot X_{C M}+O 6 \cdot X_{L M}+6 \cdot X_{A}+O 6 \cdot X_{C T}+O 6 \cdot X_{J}+6 \cdot X_{N} \leq \\
& 5 \cdot\left(A_{1} \cdot X_{C M}+A_{1} \cdot X_{L M}+A_{1} \cdot X_{A}+A_{1} \cdot X_{C T}+A_{1} \cdot X_{J}+A_{1} \cdot X_{N}\right) \\
& 4 \cdot\left(A_{2} \cdot X_{C M}+A_{2} \cdot X_{L M}+A_{2} \cdot X_{A}+A_{2} \cdot X_{C T}+A_{2} \cdot X_{J}+A_{2} \cdot X_{N}\right) \\
& +9 \cdot\left(A_{3} \cdot X_{C M}+A_{3} \cdot X_{L M}+A_{3} \cdot X_{A}+A_{3} \cdot X_{C T}+A_{3} \cdot X_{J}+A_{3} \cdot X_{N}\right) \\
& +4 \cdot\left(A_{4} \cdot X_{C M}+A_{4} \cdot X_{L M}+A_{4} \cdot X_{A}+A_{4} \cdot X_{C T}+A_{4} \cdot X_{J}+A_{4} \cdot X_{N}\right) \leq 2000 \\
& X_{C M_{i}}+X_{C M_{k}}=1, \quad X_{L M_{i}}+X_{L M_{k}}=1, \quad X_{A_{i}}+X_{A_{k}}=1, \quad \text { para } i \in \mathcal{F} \text { e } k \in \mathcal{C} \\
& X_{L T_{i}}+X_{L T_{k}}=1, \quad X_{J_{i}}+X_{J_{k}}=1, \quad X_{L N_{i}}+X_{L N_{k}}=1, \quad \text { para } i \in \mathcal{F} \text { e } k \in \mathcal{C} \\
& \sum_{k=1}^{143} X_{C M_{k}} \geq 3, \quad \sum_{k=1}^{143} X_{L M_{k}} \geq 1, \quad \sum_{k=1}^{143} X_{A_{k}} \geq 5, \\
& \sum_{k=1}^{143} X_{L T_{k}} \geq 2, \quad \sum_{k=1}^{143} X_{J_{k}} \geq 5, \quad \sum_{k=1}^{143} X_{L N_{k}} \geq 1
\end{aligned}
$$

As restrições (2) e (3) limitam inferiormente e superiormente, respectivamente, a quantidade ingerida de cada nutriente. A restrição (4) limita a quantidade de Ômega 6 em no máximo 5 vezes a quantidade de Ômega 3, procurando manter a proporção de no máximo 5:1 dessas gorduras. A restrição (5) limita a quantidade de calorias da dieta em 2000 quilocalorias. O conjunto de restrições (6) e (7) evitam que na mesma refeição sejam consumidos alimentos ricos em ferro e cálcio a fim de evitar a inibição da biodisponibilidade do ferro. As restrições (8) limitam inferiormente a quantidade de alimentos consumidos em cada refeição. O conjunto de restrições (9) diz respeito ao alimento que entrará em cada refeição da dieta. Ainda foram incluídas restrições que não permitem que alimentos semelhantes sejam incluídos na mesma refeição. Por exemplo, consumir na mesma refeição leite desnatado e leite integral, couve crua e couve refogada, dois tipos de abóboras, etc... 


\section{$5 \quad$ Resultados e Discussões}

O problema foi implementado na linguagem de programação JULIA, versão 1.1 .0 e para solucioná-lo foi utilizado o pacote JuMP (Julia for Mathematical Programming) e a biblioteca GLPK (GNU Linear Programming Kit).

Para dieta ovolactovegetariana foi obtida a seguinte composição ótima de alimentos (em porção):

- Café da manhã: pão de forma tradicional, leite de vaca integral, semente de linhaça;

- Lanche da Manhã: abacate;

- Almoço: arroz integral, batata doce cozida, agrião cru, berinjela cozida, coentro desidrato, couve manteiga crua, feijão preto, óleo de Canola, gergelim, ovo de galinha cozido.

- Lanche da tarde: banana prata e linhaça.

- Jantar: arroz integral, batata doce cozida, couve manteiga crua, abacate, feijão fradinho, óleo de soja, gergelim.

- Lanche da noite: muçarela.

A tabela 2 exibe as quantidades de nutrientes e o custo de uma dia das dietas ovolactovegetariana, lactovegetariana e vegetariana estrita.

Tabela 2: Dados referentes a dietas vegetarianas

\begin{tabular}{|l|r|r|r|}
\hline & Ovoloactoveg. & Lactoveg. & Veg. estrita \\
\hline Quant. de alimento & 22 & 26 & 29 \\
\hline Custo (R\$) & 8,78 & 8,64 & 8,68 \\
\hline O-6 (g) & 9,78 & 11,22 & 10,23 \\
\hline O-3 (g) & 2,21 & 2,26 & 2,26 \\
\hline Proteína (g) & 71,34 & 55,28 & 50,97 \\
\hline Carboidrato (g) & 245,46 & 288,22 & 309,83 \\
\hline Gordura (g) & 69,11 & 57,57 & 59,33 \\
\hline Ferro(mg) $(\mathbf{g})$ & 82,56 & 72,08 & 51,06 \\
\hline Zinco (mg) & 12,04 & 12,04 & 12,08 \\
\hline Cálcio (mg) & 1334,25 & 1116,28 & 1006,48 \\
\hline B12 (mcg) & 2,8 & 2,44 & 0 \\
\hline Vitamina C (mg) & 279,34 & 157,6 & 348,29 \\
\hline Calorias (kcal) & 1889,04 & 1910,13 & 1980,17 \\
\hline
\end{tabular}

A segunda linha da tabela exibe a quantidade de alimentos que entram em cada uma das dietas. Podemos notar que a medida que a dieta tem mais restrições, mais alimentos são necessários para atender as recomendações nutricionais e o custo mínimo.

A quantidade de proteína atende ao mínimo recomendado para o organismo nas três dietas, porém podemos observar que a medida que aumentam as restrições alimentares na dieta, diminui a quantidade de proteína, sendo que na dieta vegetariana estrita, a quantidade de proteína ficou bem próxima do mínimo recomendável, indicando que para essa dieta é preciso prestar mais atenção a esse nutriente. As quantidades de cálcio e ferro também diminuem a medida que aumentam as restrições alimentares da dieta.

A quantidade de zinco nas três dietas ficou bem próximo do limite inferior recomendável, indicando que é preciso de fato, dar uma maior antenção a alimentos que são fontes desse nutriente. 
Porém podemos observar que a quantidade de vitamina $\mathrm{C}$ é bem maior que o mínimo recomendado o que aumenta a absorção tanto do ferro quanto do zinco.

Como já era esperado, para a dieta vegetariana estrita não é possível antender a recomendação mínima de B12, sendo necessária uma suplementação.

Em relação ao custo, há pouca variação entre as três dietas e podemos observar que o custo das três dietas é acessível.

\section{Conclusão}

Nessa trabalho adaptamos o modelo clássico do problema da dieta na Programação Linear para otimizar dietas vegetarianas. O problema foi modelado como um problema de programação linear inteira, para que fosse possível incorporar restrições que levem em consideração a biodisponibilidade de nutrientes nos alimentos. Levamos em consideração quantidades diferenciadas de ingestão de nutrientes indicados para a dieta e questões relacionadas à biodisponibilidade do Ômega 3 , Ferro e Cálcio. Os resultados obtidos mostraram que é possível anteder as recomendações nutricionais de dietas vegetarianas para pessoas do sexo feminino de 19 a 50 anos, com excessão da B12 para pessoas vegetarianas estritas. Além disso, a dieta ótima nos três casos apresentam custos acessíveis.

Um trabalho futuro pode incorporar ao problema mais restrições relacionadas à biodisponibilidade, realizar a mesma análise para uma dieta de um indivíduo do sexo masculino e realizar uma comparação entre a dieta onívora e dietas vegetarianas.

\section{Referências}

[1] Brasil, Ministério da saúde, Guia alimentar para a população brasileira 2. ed., 1. reimpr. Brasília, 2014, ISBN 978-85-334-2176-9.

[2] Couceiro, P.; Slywitch, E.; Lenz, F. Boldrini, J. L., Costa, S. I. R., Ribeiro, V. R. and Wetzler, H. G. Padrão alimentar da dieta vegetariana. Rev. Einstein, vol.6, n ${ }^{o}$ 3, São Paulo, 2008.

[3] Dantzig, G. B., The Diet Problem. Interfaces, vol. 20, nº 4, pp. 43-47, 1990.

[4] Fontes, C.L.N., Escolha de um regime alimentar vegetariano - motivações e suas implicações na saúde e no meio ambiente. Dissertação de Mestrado em Economia e Gestão do Ambiente, Faculdade de economia, Universidade Porto, 2018.

[5] Institute of Medicine, Dietary Reference Intakes: The Essential Guide to Nutrient Requirements. Washington, DC: The National Academies, 2006.

[6] Key, T. J.; Fraser, G.E., Thoroggod, M.; Appleby P.N.; Beral, V.; Reeves, G; Burr, M.L., Chang-Claude, J.; Frentzel-Beyme, R.; Kuzma, J. W.; Mann J.; McPherson, K., Mortality in vegetarians and nonvegetarians: detailed findings from a collaborative analysis of 5 prospective studies. Am J Clin Nutr. pp. 516-524, 1999.

[7] Slywich, E., Guia Alimentar de Dietas Vegetarianas para Adultos, Sociedade Vegetariana Brasileira, Florianópolis, 2012.

[8] Universidade Estadual de Campinas - UNICAMP. Tabela brasileira de composição de alimentos - TACO. 4. ed. rev. e ampl. Campinas: UNICAMP/NEPA, 2011. 161 p.

[9] Van Dooren, C. A Review of the Use of Linear Programming to Optimize Diets, Nutritiously, Economicallyand Environmentally, Front. Nutr., 2018. DOI: org/10.3389/fnut.2018.00048 